MSUHEP-061231

\title{
Torsion Phenomenology at the LHC
}

\author{
A.S. Belyaev, ${ }^{1}$ I.L. Shapiro, ${ }^{2}$ and M.A.B. do Vale $^{3}$ \\ ${ }^{1}$ Department of Physics and Astronomy, \\ Michigan State University, East Lansing, MI 48824, USA* \\ ${ }^{2}$ Departamento de Física - ICE, Universidade Federal de Juiz de Fora, \\ Juiz de Fora, 36036-330, MG, Brazil 脯 \\ ${ }^{3}$ Departamento de Ciências Naturais, Universidade Federal de São João del Rei, \\ São João del Rei, 36301-160, MG, Brazil §
}

\begin{abstract}
We explore the potential of the CERN Large Hadron Collider (LHC) to test the dynamical torsion parameters. The form of the torsion action can be established from the requirements of consistency of effective quantum field theory. The most phenomenologically relevant part of the torsion tensor is dual to a massive axial vector field. This axial vector has geometric nature, that means it does not belong to any representation of the gauge group of the SM extension or GUT theory. At the same time, torsion should interact with all fermions, that opens the way for the phenomenological applications.

We demonstrate that LHC collider can establish unique constraints on the interactions between fermions and torsion field considerably exceeding present experimental lower bounds on the torsion couplings and its mass. It is also shown how possible non-universal nature of torsion couplings due to the renormalization group running between the Planck and TeV energy scales can be tested via the combined analysis of Drell-Yan and $t \bar{t}$ production processes.
\end{abstract}

\section{Contents}

\section{Introduction}

\section{The Effective Approach to Torsion}

\footnotetext{
$\dagger$ Also at Tomsk State Pedagogical University, Russia

*Electronic address: belyaev@pa.msu.edu

${ }^{\ddagger}$ Electronic address: shapiro@fisica.ufjf.br

$\S$ Electronic address: aline@ufsj.edu.br
} 
A. Limits from di-lepton production

B. Limits from $p p \rightarrow t \bar{t}$ process: the test of $\eta_{t}$ coupling

8

11

\section{Conclusions}

13

Acknowledgments.

14

\section{References}

\section{INTRODUCTION}

The Standard Model of particle physics (SM) is a very successful theory, however there is an expectation that one day we observe the signs of a new physics beyond the scope of the SM. Which kind of a new physics can be expected? Answering this question is an important issue, because the same experimental data can be always interpreted in many different ways and only consistent theoretical consideration can provide a reasonable perspective for a phenomenological interpretation of experimental data. From the theoretical viewpoint, one of the main shortcomings of the Standard Model and its natural (non-minimal) extensions is their inability to incorporate quantum gravity. The natural conclusion is that, if the consistent quantum theory of all four fundamental interactions is really possible, it should include certain extension of the known physics, that is SM plus General Relativity(GR). Modifications should be expected, at the first place, in the gravitational sector of the theory.

The most realistic candidate to be the universal theory of everything is the (super)string theory. The merit of this theory (along with very important advances in formal purely theoretical developments) is the possibility to incorporate gravity into the scheme of unification. All fundamental interactions emerge as low-energy manifestations of the fundamental quantum object - (super)string. The success of the superstring program produced a variety of mathematically perfect versions of this theory [1, 2, 3]. Unfortunately there is no real perspective for any existing or projected experiment which could give an answer which one of these mathematically perfect theories is actually correct. The situation becomes even more complicated if we remember that the consistent theory of superstring can be formulated only in higher dimensions and that extra dimensions may be compactified in many different ways. Moreover at any finite order of the string perturbation theory there is an additional ambiguity of defining the parametrization of external background fields [4, 5, 6] (see also detailed discussion of this issue in [7]).

In this situation it is completely justified to apply a phenomenological approach and simply consider most natural extensions of GR assuming they might come from the unknown fundamental theory. The remarkable examples of such an approach are extra dimensions leading to TeV scale gravity [8] and Lorentz and CPT violations [9]. In this paper we shall concentrate on the phenomenological aspects of torsion gravity theory, which is traditionally 
considered as one of the most natural extensions of GR [7, 10]. Let us notice that global torsion field is in fact one of the main candidates for the Lorentz and CPT violation parameters. Such field can be a component of vacuum resulting from some new symmetry breaking phenomenon [7, 12]. Another possibility is to treat torsion as fundamental propagating field, which has well defined action and is characterized by such parameters as torsion mass and the values of the coupling between torsion and fermions (quarks and leptons). In the framework of effective quantum theory one faces rigid restrictions on the torsion parameters, originating from both phenomenological and theoretical analysis [13, 14, 15, 16, 17]. In the present paper we study the LHC potential to improve limits on the torsion parameter space which we have derived earlier from LEP and Tevatron data [13].

The torsion field $T_{\beta \gamma}^{\alpha}$ is defined as follows ${ }^{1}$ :

$$
\Gamma_{\beta \gamma}^{\alpha}-\Gamma_{\gamma \beta}^{\alpha}=T_{\beta \gamma}^{\alpha} .
$$

It proves useful to divide torsion into irreducible components

$$
T_{\alpha \beta \mu}=\frac{1}{3}\left(T_{\beta} g_{\alpha \mu}-T_{\mu} g_{\alpha \beta}\right)-\frac{1}{6} \varepsilon_{\alpha \beta \mu \nu} S^{\nu}+q_{\alpha \beta \mu},
$$

where the axial vector $S^{\mu}$ is dual to the completely antisymmetric torsion tensor, $T_{\alpha}$ is a vector trace of torsion and $q_{\alpha \beta \mu}$ is a tensor which satisfies the constraints $q_{\cdot \beta \alpha}^{\alpha}=0$ and $q_{\alpha \beta \mu} \varepsilon^{\alpha \beta \mu \nu}=0$.

The general nonminimal action of a Dirac fermion coupled to torsion has the form

$$
S_{f}=\int \sqrt{g}\left\{i \bar{\psi} \gamma^{\mu}\left(\nabla_{\mu}-i \eta_{1} \gamma^{5} S_{\mu}+i \eta_{2} T_{\mu}\right) \psi-m \bar{\psi} \psi\right\}
$$

where $\eta_{1}, \eta_{2}$ are nonminimal parameters and $\nabla_{\mu}$ is Riemannian covariant derivative (without torsion). Let us remember that the minimal interaction corresponds to the action

$$
S_{m i n, f}=\frac{\imath}{2} \int d^{4} x \sqrt{-g}\left\{\bar{\psi} \gamma^{\mu} \tilde{\nabla}_{\mu} \psi-\tilde{\nabla}{ }_{\mu} \bar{\psi} \gamma^{\mu} \psi+2 \imath m \bar{\psi} \psi\right\}
$$

where $\tilde{\nabla}_{\mu}$ is the covariant derivative with torsion. Direct calculation shows that this expression corresponds to the values $\eta_{1}=-1 / 8, \eta_{2}=0$ of parameters in (21). It has been shown long ago [18] that the quantum theory is not renormalizable for a fixed non-zero value of $\eta_{1}=\eta$, while the absence of $\eta_{2}$ does not imply any special difficulties. Therefore, in what follows we consider $\eta_{1}$ as an arbitrary parameter and take $\eta_{2}=0$ for simplicity. This is equivalent to consider the completely antisymmetric torsion tensor $T_{\alpha \beta \gamma}=-\frac{1}{6} \varepsilon_{\alpha \beta \gamma \mu} S^{\mu}$. So, we shall choose the following action for each of the fermions $\psi_{(i)}$ :

$$
\mathcal{S}_{\text {non-min }}^{T S-\text { matter }}=i \int d^{4} x \sqrt{g} \bar{\psi}_{(i)}\left(\gamma^{\alpha} \nabla_{\alpha}+\imath \eta_{i} \gamma^{5} \gamma^{\mu} S_{\mu}-\imath m_{i}\right) \psi_{(i)},
$$

\footnotetext{
${ }^{1}$ See [10] and [7] for different introductions to torsion
} 
where $\eta_{(i)}$ is the non-minimal interaction parameter for the corresponding spinor. Let us remember that torsion is a tensor which does not depend on metric. One can always assume, for simplicity, that the metric is flat $g_{\alpha \beta}=\eta_{\alpha \beta}$ and therefore the torsionless part of the covariant derivative is nothing else but a partial derivative $\nabla_{\alpha}=\partial_{\alpha}$.

The action for the propagating torsion can be established using the consistency criteria for the effective low-energy quantum theory of torsion coupled to the gauge model with fermions and scalars, such as the Standard Model. Requesting unitarity and renormalizability of the theory in the low-energy sector one arrives at the unique possible form of the torsion action [13]

$$
\mathcal{S}_{\text {tor }}^{T S-k i n}=\int d^{4} x\left\{-\frac{1}{4} S_{\mu \nu} S^{\mu \nu}+\frac{1}{2} M_{T S}^{2} S_{\mu} S^{\mu}\right\}
$$

where $M_{T S}$ is the mass of the torsion and $S_{\mu \nu}=\partial_{\mu} S_{\nu}-\partial_{\nu} S_{\mu}$. The consistency of the theory based on the action (5) holds when the quantum effects of the fermion loops are taken into account. After that point one can perform both theoretical and phenomenological analysis ${ }^{2}$.

The first constraints on the dynamical torsion parameters have been established in [13] based on LEP1.5 and Tevatron data for the theory with the action (5). These constraints have been updated in later paper [17] using improved LEP2 and Tevatron statistics. In [15] constraints for the similar theory with extra dimensions have been presented. Furthermore, other phenomenological and theoretical aspects of torsion, in the framework of high energy theory, have been explored in [21] and [22].

The theoretical investigation which is the most important for us has been performed in [13, 14], where the consistency of the effective theory with quantized torsion was studied. Following the standard approach, we postulate that all fields have to be quantized in the framework of effective approach, and found that, for the torsion-fermion system this can be consistently done only if the constraint $M_{T S} / \eta_{i} \gg m_{i}$ ( or, equivalently, $M_{T S} \gg \eta_{i} \times m_{i}$ ) is satisfied for all fermions with the masses $m_{i}$ and with the nonminimal parameter $\eta_{i}$. Despite this restriction does not rule out the existence of torsion, it puts a strong constraint either on its propagation or on its interactions with fermions. Together with the phenomenological bounds for the two parameters $M_{T S}$ and $\eta$ [13] we arrive at the very strong constraints on the torsion parameters. The purpose of the present article is to study the LHC potential to put further constraints on parameters $M_{T S}$ and $\eta_{i}$. As we show, the limits on the $\left(M_{T S}, \eta\right)$ parameter space which can be obtained at the LHC, are much more restrictive than the ones obtained previously using LEP and TEVATRON data [13].

The paper is organized as follows. In the next section we shall briefly discuss the theoretical background of effective quantum theory of torsion. In this part we assume the reader can consult the previous publications [7, 13, 14, 19] for more detailed and pedagogical introduc-

\footnotetext{
${ }^{2}$ Similar phenomenological analysis based on the longitudinal torsion has been performed in [20]. However the longitudinal torsion is inconsistent from the formal viewpoint.
} 
tion. Furthermore, we shall calculate the approximate relations between the parameters $\eta_{i}$ for different kinds of fermions $i$, using renormalization group and a very natural assumption of equal $\eta_{i}$ at the Planck scale, where all fields should be generated and start to interact with the geometry described, in particular, by torsion. In section 3 we shall discuss the phenomenology of torsion for $p p \rightarrow \ell \ell$ and $p p \rightarrow t \bar{t}$ processes at the LHC and compare the corresponding upper bounds for the torsion parameters $M_{T S}$ and $\eta$ with the ones for obtained previously from LEP and Tevatron. Finally, in section 4 we draw our conclusions.

\section{THE EFFECTIVE APPROACH TO TORSION}

The considerations which lead to the antisymmetric torsion action (5) look as follows. As far as torsion is considered as a dynamical field, one has to incorporate it into the SM along with other vector fields. Due to the assumed geometric (gravitational) nature of torsion, it is coupled to all fermionic fields. The action of these fields looks as (4) with additional SM-type interaction to the gauge and scalars fields.

The fermionic sector of the gauge theory consists of the actions (2) supplemented by the gauge and Yukawa interactions. The symmetries of the theory include usual gauge transformations and an extra transformation related to torsion

$$
\psi \rightarrow \psi^{\prime}=\psi e^{\gamma_{5} \beta(x)}, \quad \bar{\psi} \rightarrow \bar{\psi}^{\prime}=\bar{\psi} e^{\gamma_{5} \beta(x)}, \quad S_{\mu} \rightarrow S_{\mu}^{\prime}=S_{\mu}-\eta^{-1} \partial_{\mu} \beta(x) .
$$

Massive fermion term is not invariant under the last transformation, so it corresponds to the softly broken symmetry. This symmetry has a significant impact on the renormalization structure of the theory. Since it is softly broken, it does not forbid massive counterterms in the torsion sector. Therefore, $S_{\mu}$ has to be a massive field, for otherwise one can not control quantum corrections to the corresponding massive term. Furthermore, the renormalization in the massless sector does not depend on the massive parameters. If we start from the most general action with second derivatives for $S_{\mu}$,

$$
S_{\text {tor }}=\int d^{4} x\left\{-a S_{\mu \nu} S^{\mu \nu}+b\left(\partial_{\mu} S^{\mu}\right)^{2}+\frac{1}{2} M_{t s}^{2} S_{\mu} S^{\mu}\right\},
$$

an extra symmetry (6) tells us that the $S_{\mu \nu} S^{\mu \nu}$-type counterterms really takes place. Direct calculations also confirm this fact and hence the $-a S_{\mu \nu} S^{\mu \nu}$-term is necessary in the consistent theory for the same reason as the massive term. If we do not introduce the transverse kinetic term, it will emerge at quantum level anyway. Furthermore, the co-existence of the $-a S_{\mu \nu} S^{\mu \nu}$-term and of the $b\left(\partial_{\mu} S^{\mu}\right)^{2}$-term violate unitarity of the theory. In this way we arrive to the unique form of the torsion action (5).

The high energy phenomenology of the torsion theory (5) is based on the fact the axial vector $S_{\mu}$ has no direct analogs in the Standard Model. At the first place, this is a massive axial vector and, also, it is not related to a gauge group of the theory and interacts with the 
matter fields only via the $\eta$-coupling in the Eq. (4). At the same time, this interaction is not necessarily universal since the values of parameters $\eta_{i}$ may be different for distinct fermions. The renormalization group equations for different $\eta_{i}$ are dependent on the corresponding Yukawa couplings [13, 18]. As far as these couplings are different, the renormalization group equations for different $\eta_{i}$ are also different and finally the values of these parameters at the low-energy scale should be also different.

The renormalization group equations for $\eta_{i}$ have been obtained and discussed in [13] in a rather general form. In order to evaluate the values of $\eta_{i}$ at the given scale we shall assume that (as it was already mentioned before) the values of all $\eta_{i}$ are equal at the Planck scale. In this case the set of the values of the nonminimal parameters $\eta_{i}\left(\mu_{L H C}\right)$ at the energy scale available in LHC experiments should result from the renormalization group flow for these parameters. Let us start from the simple version of the renormalization group equations $^{3}$

$$
(4 \pi)^{2} \mu \frac{d \eta_{i}(\mu)}{d \mu}=\frac{1}{3} \eta_{i}^{3}(\mu)+C h_{i}(\mu) \eta_{i}(\mu) .
$$

The solution of this equation is simple provided we know the function $h_{i}(\mu)$, but in fact this function is available only for relatively low energies where the SM can be experimentally verified. At higher energy scale one needs to know a corresponding gauge model (being it some GUT or supersymmetric extension of the SM or something else, e.g. just a proper SM valid until the Planck scale). Of course, this information is not available. However, as we shall see in a moment, one can obtain the relevant data even without it.

Let us start from the minimal assumptions that the effective Yukawa coupling are constants which possess the same values as all scales. Furthermore we assume a very small $\eta_{i}$, such that only the second term in the Eq. (8) is relevant. Then the solution of the above equation becomes very simple

$$
\eta_{i}(\mu)=\eta_{i}\left(\mu_{0}\right)\left(\frac{\mu}{\mu_{0}}\right)^{C h_{i} /(4 \pi)^{2}} .
$$

Our working hypothesis is that the nonminimal parameters are $\eta_{i}\left(\mu_{0}\right) \equiv \eta_{\text {Planck }}$ equal at the fundamental Planck scale $\mu_{0}=M_{P}=10^{19} \mathrm{GeV}$. Then the values of $\eta_{i}(\mu)$ depend only on the expression $C h_{i} /(4 \pi)^{2}$. Let us take an extreme case of the top quark, when $h_{i} \sim 1$ and assume, as it was already discussed before, that this value does not depend on scale. The coefficient $C$ has been derived for various models based on different gauge groups [18, 19]. A very optimistic estimate is $C=5$, that requires a sufficiently large gauge group of GUT. Then we obtain, for $\mu_{0}=M_{P}=10^{19} \mathrm{GeV}$ and $\mu=10^{3} \mathrm{GeV}$, the estimate

$$
\left(\frac{\mu}{\mu_{0}}\right)^{C h_{i} /(4 \pi)^{2}} \approx 10^{-1 / 2}
$$

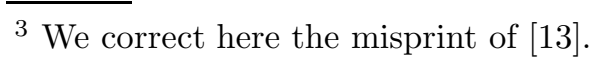


Obviously, due to our estimate $h_{i} \sim 1$, this result has relation only to the top quark case, while for other fermions the ratio will be very close to unity. Now, let us notice that in all our simplifications we have always made a choice in such a way that the ratio in (10) was becoming bigger. For instance, already for SM the value of $C$ is much smaller than 5 , the running of the Yukawa constants, being in accordance with the asymptotic freedom, reduces the effect and finally taking into account the nonlinear term in Eq.(8) is also decreasing the ratio in (10). All in all, we conclude that, except the case of the top quark, the values of all $\eta_{i}$ at the $\mathrm{TeV}$ scale must be equal or at least very close to each other, in fact they are simply equal to the universal value $\eta_{\text {Planck }}$. The top quark parameter $\eta_{t}$ may be, in principle, different from the others because of the potentially stronger running between the Planck and Fermi (or TeV) scales. The lesson we learned here is that the list of unknown parameters of the theory includes the mass of the torsion $M_{t s}$ plus the universal nonminimal parameters $\eta_{i \neq t} \equiv \eta$ and also $\eta_{t}$ which may be a bit smaller than $\eta$. In the previous papers on the subject [13] we did not take the possible difference between $\eta$ and $\eta_{t}$ into account.

In the present case we are going to take into account the processes involving the top quark and also the ones involving other fermionic particles. Our purpose is to improve the upper bound for the torsion parameters and therefore it is reasonable to account for the possible difference between $\eta_{t}$ and $\eta_{i \neq t} \equiv \eta$.

\section{PHENOMENOLOGICAL CONSEQUENCES AT LHC}

The CERN Large Hadron Collider (LHC) in about one year will start proton-proton collisions at $14 \mathrm{TeV}$ in the center of mass energy. Thanks to its unprecedented energy and luminosity, the LHC will be able to probe the new physics at the several $\mathrm{TeV}$ scale.

The straightforward consequence of the heavy torsion interacting with fermion fields is the effective four-fermion contact interaction of leptons and quarks (see details in Ref. [13]). Since torsion interacts universally with light quarks and leptons, the most sensitive process to probe torsion interactions with light fermions is the Drell-Yan (DY) process of dilepton production. It has been shown in ATLAS and CMS collaborations studies that compositeness scale at the LHC can be probed down to $20-30 \mathrm{TeV}$ scale via DY process [23, 24]. On the other hand, the $p p \rightarrow t \bar{t}$ process is the natural choice to probe torsion coupling with top-quark.

To perform our analysis we have implemented interactions of torsion with fermions described by Eq. 4 into CalcHEP package [25]. The study has been done at the parton-level using CalcHEP 2.45 together with the realistic experimental efficiencies. The CTEQ6M parton distribution function (PDF) with the QCD renormalization and factorization scales equal to the torsion mass $M_{T S}$ was used in CalcHEP. In our analysis we assume a total integrated luminosity of $100 \mathrm{fb}^{-1}$. 


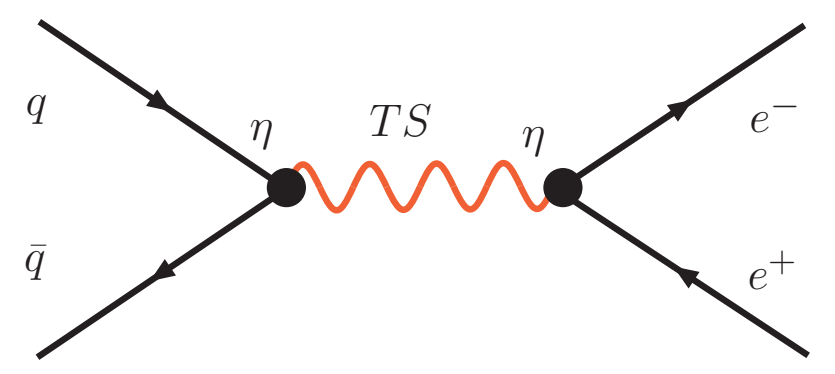

FIG. 1: Additional Feynman Diagram for process $p p \longrightarrow e^{+} e^{-}$at LHC introduced by the torsion (TS) interactions.

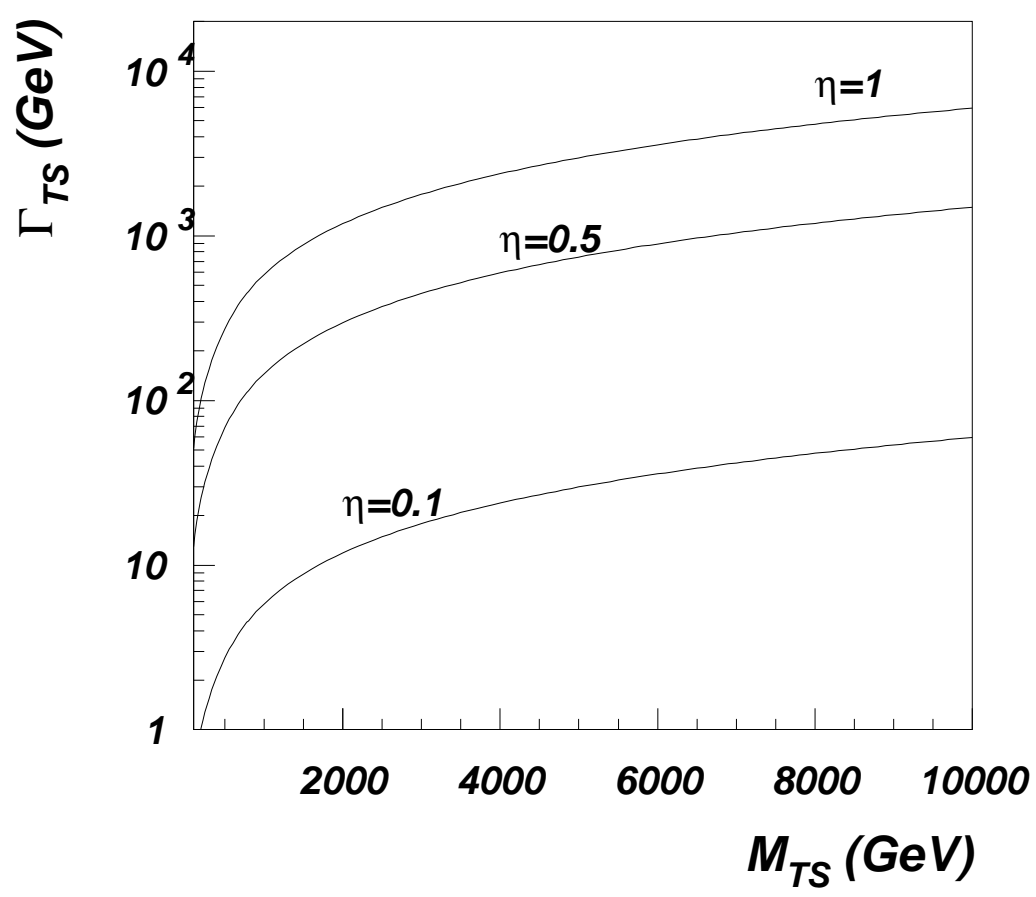

FIG. 2: Torsion width versus its mass for various values of $\eta$ coupling under assumption $\eta=\eta_{t o p}$

\section{A. Limits from di-lepton production}

In Fig. 1 we present Feynman diagram for torsion contribution to di-lepton production. One should note that heavy torsion can not be exactly approximated by four-fermion contact interactions in general, since it has a significant width, as one can see from Fig. 2. For small values of $\eta$ coupling resulting to $M_{T S} \gg \Gamma_{T S}$ four-fermion contact interaction would be a good approximation for $\hat{S}<M_{T S}$. In our studies we use exact interaction described by Eq. (4) and do not use approximation of the contact interaction of torsion. In this way we take into account both cases - when torsion is being produced either on-shell or off-shell. The cross section of torsion production rapidly drops down to about $10^{-1} \mathrm{fb}$ with the increasing 


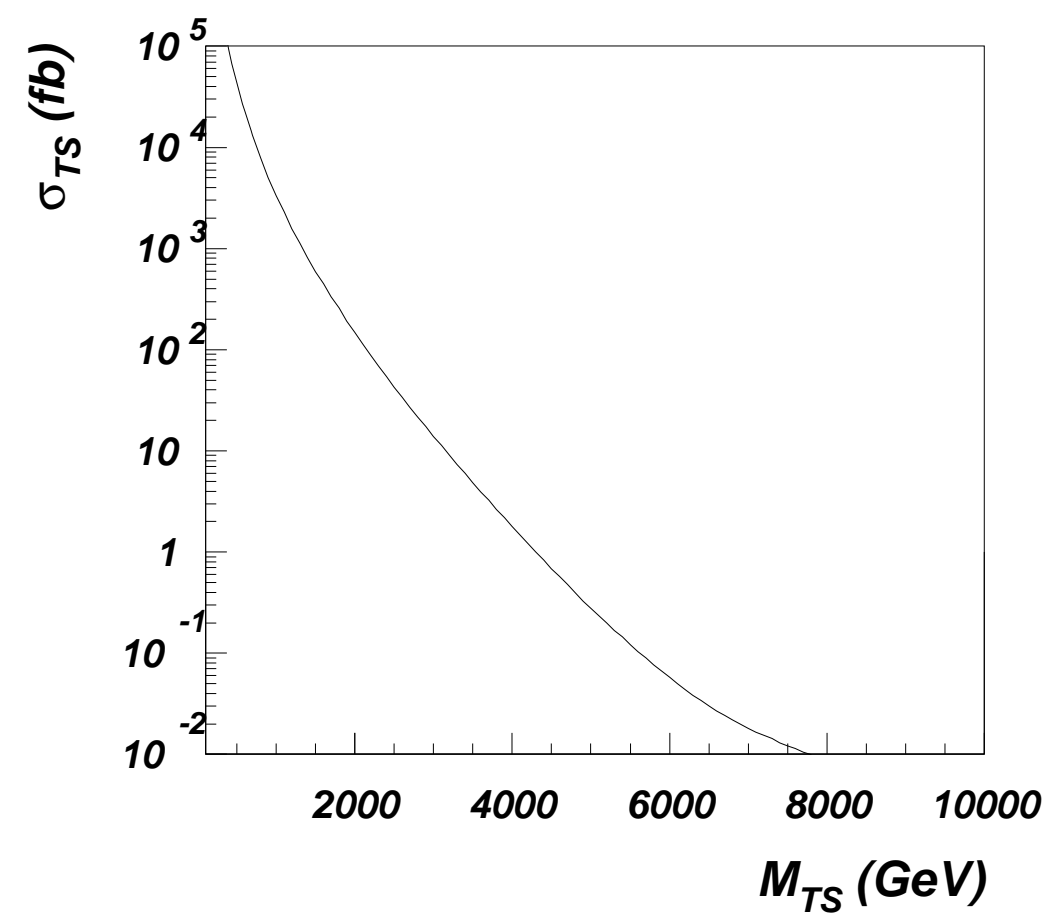

FIG. 3: Torsion production cross section at the LHC for $\eta=0.1$.

of its mass up to about $5 \mathrm{TeV}$ for $\eta=0.1$ as one can see in Fig. 3. For this cross section one could expect about 10 events from the torsion which would be observable at the LHC in case if background reduced down enough with the appropriate choice of the kinematical cuts. The cross section of the torsion produced on-shell scales as $\eta^{2}$ and as $\eta^{4}$ in case of the off-shell torsion production.

To take into account the detector acceptance we require the rapidity of the electron and the positron to be between -2.5 and 2.5. We have also found that the cut $p_{T}^{e}>M_{T S} / 3$ optimizes the signal significance together with the mass window cut on the $e^{+} e^{-}$invariant mass: $\left|M_{T S}-M_{e^{+} e^{-}}\right|<2 \times \Gamma_{T S}$, where $\Gamma_{T S}$ is the torsion width.

In our study we require signal-to-background ratio $(\mathrm{S} / \mathrm{B})$ to be bigger than $1 / 2$ which happens to be always the case after we apply kinematical cuts above. This S/B ratio cut was applied to ensure the signal observation in the presence of the possibly large ( $50 \%)$ uncertainty in the parton density function (PDF) in the high $x$ region of the heavy torsion production. The $P_{T}$ and mass window cut which we have chosen actually provide good S/B ration above $1 / 2$ and very effective background suppression while leaving intact more than $50 \%$ of the signal events.

We have calculated the significance $(S / \sqrt{B})$ from the signal+background $(\mathrm{S}+\mathrm{B})$ events excess over the background $(\mathrm{B})$. Under $\mathrm{S}+\mathrm{B}$ we understand the rate of the signal+background including the effect of the signal and background interference i.e. interfer- 


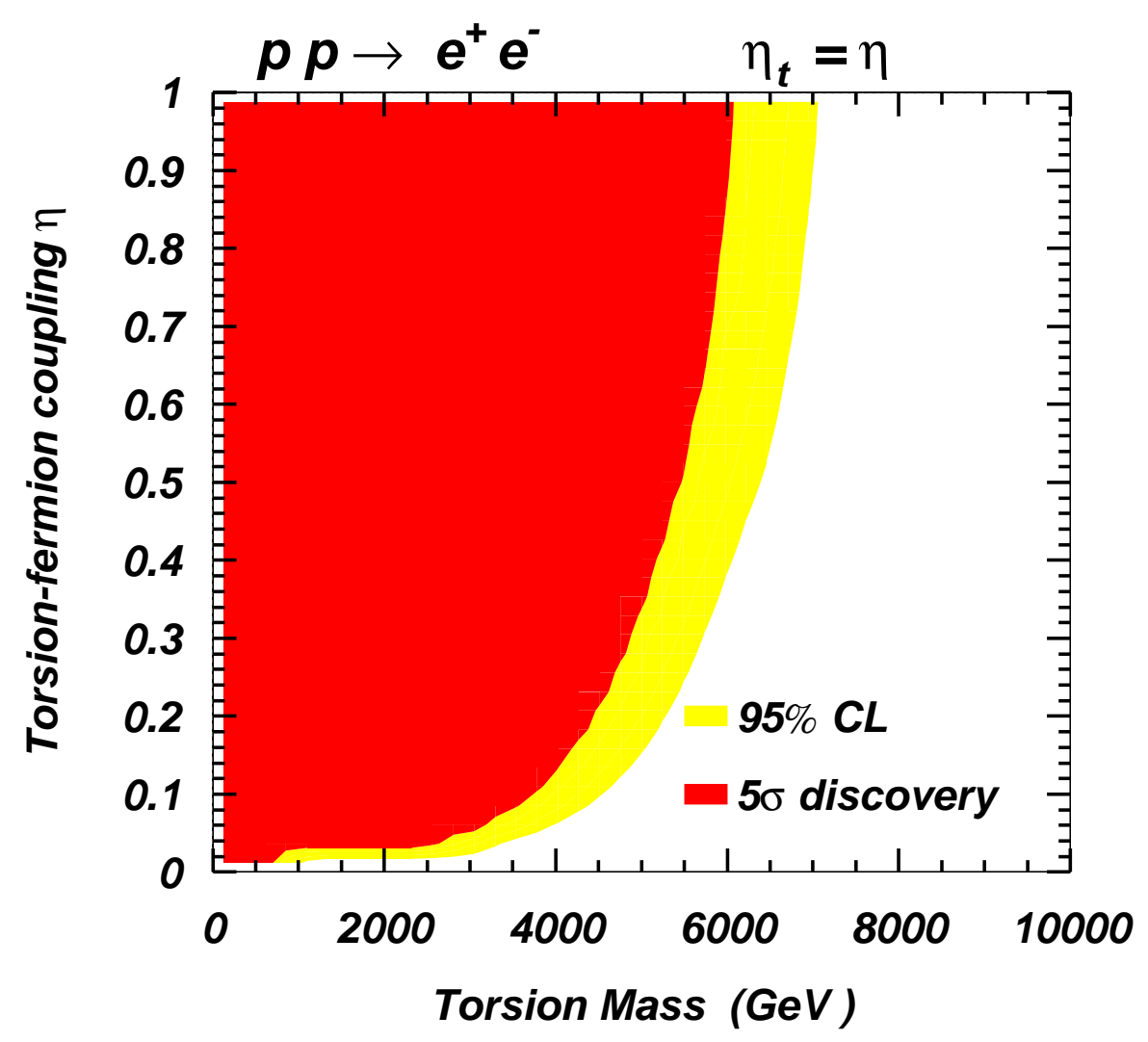

FIG. 4: The LHC discovery reach and 95\% CL exclusion bounds from $p p \rightarrow e^{+} e^{-}$process for the $\left(M_{T S}, \eta\right)$ parameter space.

ence between $\gamma / Z$-boson and the torsion exchange. In the region of low statistics when the number of signal events drops below 30, we have used Poisson statistics formula for significance calculation.

The LHC discovery reach and 95\% CL exclusion bounds from $p p \rightarrow e^{+} e^{-}$process for the $\left(M_{T S}, \eta\right)$ parameter space are shown in Fig. 4. One can see that for $\eta=0.1$, LHC can exclude $M_{T S} \lesssim 4.5 \mathrm{TeV}$ at $95 \% \mathrm{CL}$ while torsion with about $3.5 \mathrm{TeV}$ mass can be discovered for this value of $\eta$. For $\eta=1 \mathrm{LHC}$ is sensitive to about $7 \mathrm{TeV}$ torsion at 95\%CL while torsion with about $6 \mathrm{TeV}$ mass can be discovered.

Comparing with previous constraints from Tevatron and LEP on the torsion parameters [13] (Fig. 5) (see also [17] for the improved LEP2 and Tevatron bounds and Fig.5 in there) we can see that indeed LHC is much more sensitive to $\left(M_{T S}, \eta\right)$ parameter space. For a given value of $\eta$, LHC could extend the torsion mass reach by about one order of magnitude from di-lepton production. Moreover, the more sophisticated analysis and combination of electron and muon channels could improve further the LHC reach for the torsion parameter space. 

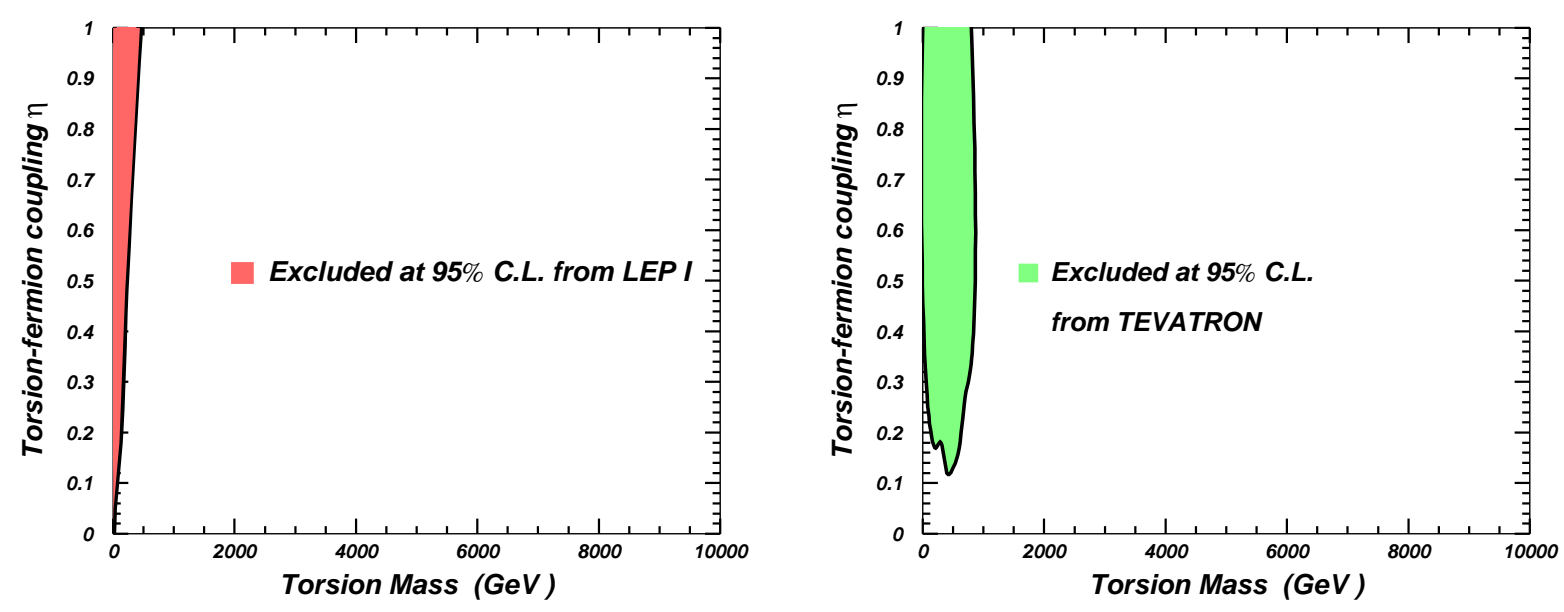

FIG. 5: Bounds for the $\left(M_{T S}, \eta\right)$ parameter space at 95\% C.L. obtained from from LEP data [13] (left) and TEVATRON data [13] (right).

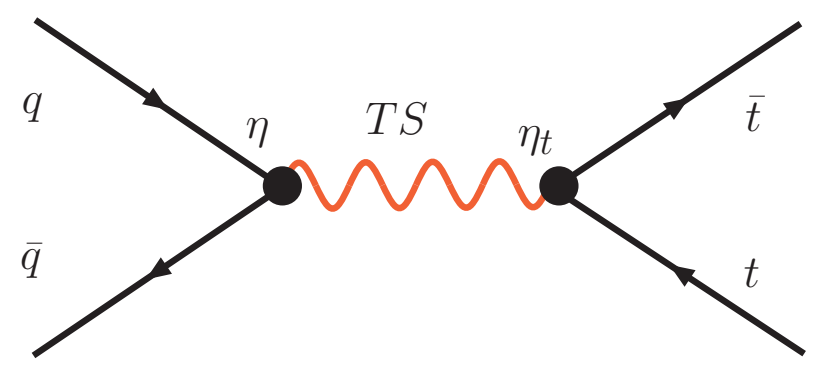

FIG. 6: Additional Feynman Diagram for process $p p \longrightarrow t \bar{t}$ at LHC introduced by the torsion interactions.

\section{B. Limits from $p p \rightarrow t \bar{t}$ process: the test of $\eta_{t}$ coupling}

As we pointed out in Section III, the $\eta_{t}$ coupling of torsion to top-quark may not be necessarily universal as compared to torsion couplings to the light fermions.

Since this coupling is the new additional parameter in low energy theory, it should be tested on the same foot as the other parameters of the model. Eventually, the $p p \rightarrow t \bar{t}$ process is the naturally best choice to probe torsion coupling to the top-quark. In Fig. 6 we present Feynman diagram for $p p \longrightarrow t \bar{t}$ process at LHC due to the torsion exchange.

Top-quark undergoes subsequent decay chains and should be reconstructed. To keep the parton-level spirit of this study and correctly take into account qualitative experimental effects, we have utilized the $1 \%$ efficiency of the reconstruction of the $t \bar{t}$ pair. This efficiency has been obtained in the recent studies (see [26] and reference [13] therein) for the leptonhadron decay signature of $t \bar{t}$ pair. 

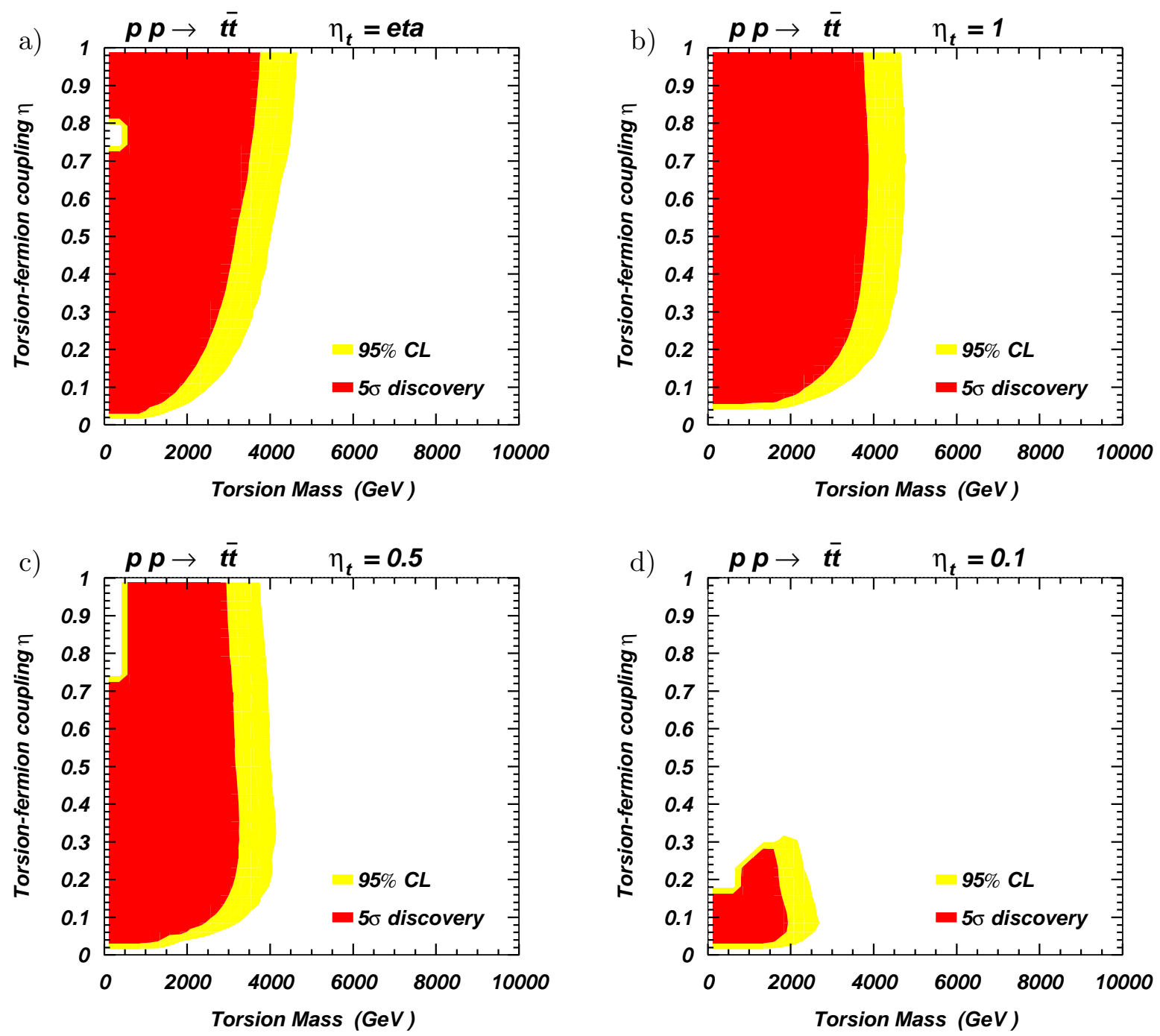

FIG. 7: LHC sensitivity to $\left(M_{T S}, \eta\right)$ parameter space for various cases of $\eta_{t}$ parameter: $\eta_{t}=\eta$, $\eta_{t}=1, \eta_{t}=0.5$ and $\eta_{t}=0.1$.

Similar to $p p \rightarrow e^{+} e^{-}$process we use $p_{T}^{t}>M_{T S} / 3$ and $\left|M_{T S}-M_{t \bar{t}}\right|<2 \times \Gamma_{T S}$ kinematical cuts on the reconstructed top-quarks to optimize the signal significance. As a result of the analysis of $p p \rightarrow t \bar{t}$ processes in Fig. 7 we present LHC sensitivity to $\left(M_{T S}, \eta\right)$ parameter space for various choices of $\eta_{t}$ parameter: $\eta_{t}=\eta, \eta_{t}=1, \eta_{t}=0.5$ and $\eta_{t}=0.1$.

One can see that the sensitivity of the LHC to torsion parameter space for $p p \rightarrow t \bar{t}$ process is worse as compared to $p p \rightarrow e^{+} e^{-}$. It is not surprising, since there are two main factors which cause this reduction:

1) the top-quark pair reconstruction efficiency is only $1 \%$;

2) the relative $t \bar{t}$ QCD background originating from the strong interactions is bigger as compared to the electroweak background for di-lepton production.

As a result, we have limited sensitivity of the LHC to the torsion parameters via $p p \rightarrow t \bar{t}$ process. For example, for $\eta \simeq \eta_{t}=0.1$ case (Fig. [7) the $5 \sigma$ sensitivity of LHC to $M_{T S}$ for 
$p p \rightarrow t \bar{t}$ process is reduced down to about $2 \mathrm{TeV}$ (as compared to $3.5 \mathrm{TeV} M_{T S}$ for $5 \sigma \mathrm{LHC}$ for $p p \rightarrow e^{+} e^{-}$process, see Fig. (4). Nevertheless, the $p p \rightarrow t \bar{t}$ process provides a unique opportunity to test the specific $\eta_{t}$ coupling. Further comments on Fig. 7 are in order. In case of $\eta_{t}<\eta$ as one can see in Fig. [7 7 ,d the LHC sensitivity to the torsion parameter space is reduced even more. This happens for the following reason. The rate of $t \bar{t}$ signature from torsion production does not change with the increase of $\eta$ while $\eta_{t}$ is constant, since the increase of the production is compensated by the decrease of $\operatorname{Br}(T S \rightarrow t \bar{t})$. In the same time the width of the torsion got increased. This increase of the torsion width causes the reduction of the signal significance and the respective reduction of the LHC sensitivity to the torsion parameters space. One can see this clearly in Fig. $7 \mathrm{~d}$ demonstrating the absence of the LHC sensitivity to torsion parameter space for $\eta \gtrsim 0.3$ and $\eta_{t}=0.1$. Contrary, for $\eta_{t}>\eta$ case (Fig. 7b) the sensitivity of the LHC to the torsion parameter space is increased.

\section{CONCLUSIONS}

We have found that LHC collider can establish unique constraints on the interactions between fermions and torsion field considerably exceeding present experimental bounds.

Due to the renormalization group running, the universal interaction between all fermions and torsion at the Planck scale may be, in principle, characterized by the different values of the fermion-torsion couplings at the $\mathrm{TeV}$ scale. This effect should take place for all kinds of fermions. However, the numerical effect of the running is negligible for all known fermions except the top quark. Therefore, the parameter space of the torsion-fermion interactions is effectively reduced to the 3-dimensional one, namely $\left(M_{T S}, \eta, \eta_{t}\right)$. Our phenomenological analysis has shown that this parameter space can be severely constrained with Drell-Yan and $t \bar{t}$ processes at the LHC. Moreover, for the first time we have demonstrated how possible nonuniversal nature of torsion-fermion interactions can be tested via the combined analysis of Drell-Yan and $t \bar{t}$ processes. The results are summarized in Fig. 4 and Fig. 7 demonstrating that LHC can improve present bounds on the torsion parameters by about one order of magnitude. For example, for $\eta=0.1, M_{T S} \lesssim 4.5 \mathrm{TeV}$ can be excluded at 95\% CL. However the test of the non-universal nature of $\eta \simeq \eta_{t} \simeq 0.1$ requires $M_{T S} \simeq 2 \mathrm{TeV}$ which provides high enough statistics for both $p p \rightarrow T S \rightarrow \ell^{+} \ell^{-}$and $p p \rightarrow T S \rightarrow \overline{t t}$ processes.

We have also shown that, since torsion width can be very large, the four-fermion contact interaction approach used previously in several publications, could be not a good approximation for the investigation of the torsion phenomenology. Finally, we would like to mention that there is a room for more sophisticated analysis and combination of electron and muon channels to improve further the LHC reach for the torsion parameter space. Also, more sophisticated analysis with full detector simulation of the $p p \rightarrow t \bar{t}$ process and $t \bar{t}$ pair reconstruction are necessary to understand more precisely the LHC sensitivity to non-universal nature of $\eta$ and $\eta_{t}$ couplings. We consider this paper as the first step in bringing ideas on 
the torsion phenomenology at the LHC to HEP community to stimulate further studies at the LHC.

\section{Acknowledgments.}

The work of M.A.B. do Vale and I.Sh. has been partially supported by the PRONEX project, research grants from FAPEMIG (MG, Brazil) and CNPq (Brazil). Besides, I.Sh. was supported by the Senior Associated Membership Grant from ICTP (Italy). The work of A.B. was supported by the US National Science Foundation under award PHY-0555545. I.Sh. is also grateful to the Theory Division of CERN and Department of Theoretical Physics at the University of Zaragoza for kind hospitality.

[1] M.B. Green, J.H. Schwarz and E. Witten, Superstring Theory (Cambridge University Press, Cambridge, 1987).

[2] J. Polchinsky, String Theory, (Cambridge University Press, Cambridge, 1987).

[3] S.V. Ketov, Quantum Non-Linear Sigma Models : From Quantum Field Theory to Supersymmetry, Conformal Field Theory, Black Holes, and Strings. (Springer Verlag, 2000).

[4] B. Zwiebach, Phys. Lett. 156B (1985) 315.

[5] S. Deser and A.N. Redlich, Phys. Lett. 176B (1986) 350.

[6] A.A. Tseytlin, Phys. Lett. 176B (1986) 92.

[7] I.L. Shapiro, Phys. Repts., 357 (2002) 113.

[8] N. Arkani-Hamed, S. Dimopoulos and G.R. Dvali, Phys. Lett. B429 (1998) 263

I. Antoniadis, N. Arkani-Hamed, S. Dimopoulos and G.R. Dvali, Phys. Lett. B436 (1998) 257.

[9] V.A. Kostelecky, S. Samuel, Phys. Rev. D39 (1989) 683;

For the recent review see, e.g. R. Bluhm, Overview of the SME: Implications and phenomenology of Lorentz violation, hep-ph/0506054.

[10] F.W. Hehl, Gen. Relat. Grav. 4 (1973) 333; 5 (1974) 491;

F.W. Hehl, P. Heide, G.D. Kerlick and J.M. Nester, Rev. Mod. Phys.48 (1976) 393; see also 11] for the review of geometric aspects of torsion theories.

[11] F. W. Hehl, J.D. McCrea, E.W. Mielke and Yu. Neeman, Phys. Rep. 258 (1995) 1-171.

[12] V.A. Kostelecky, Phys. Rev. D69 (2004) 105009.

[13] A.S. Belyaev and I.L. Shapiro, Phys.Lett. 425B(1998)246; Nucl.Phys. B543(1999)20.

[14] G. de Berredo-Peixoto, J.A. Helayel-Neto and I. L. Shapiro, JHEP 02 (2000) 003.

[15] L.N. Chang, O. Lebedev, W. Loinaz and T. Takeuchi, Phys.Rev.Lett. 85 (2000) 3765;

O. Lebedev, Phys. Rev. 65D (2002) 124008.

[16] P. Das, U. Mahanta and S. Raychaudhuri, arXiv:hep-ph/0211137.

[17] U. Mahanta and S. Raychaudhuri, arXiv:hep-ph/0307350. 
[18] I.L. Buchbinder and I.L. Shapiro, Phys. Lett. 151B (1985) 263;

I.L. Buchbinder and I.L. Shapiro, Class. Quant. Gravity. 7 (1990) 1197, see also [19].

[19] I.L. Buchbinder, S.D. Odintsov and I.L. Shapiro, Effective Action in Quantum Gravity (IOP Publishing, Bristol, 1992).

[20] S.M. Carroll and G.B. Field, Phys.Rev. 50D (1994) 3867.

[21] M. Alimohammadi and A. Shariati, Eur. Phys. J. 21C (2001) 193.

[22] L. Freidel, D. Minic and T. Takeuchi, Phys. Rev. 72D (2005) 104002.

[23] "ATLAS detector and physics performance. Technical design report. Vol. 2"," CERN-LHCC99-15

[24] “ CMS Physics Technical Design Report Volume II: Physics Performance ”, CERN/LHCC 2006-021

[25] A. Pukhov, arXiv:hep-ph/0412191.

[26] K. Agashe, A. Belyaev, T. Krupovnickas, G. Perez and J. Virzi, arXiv:hep-ph/0612015. 